\title{
A TOOL FOR EMOTIONAL USER EXPERIENCE ASSESSMENT OF WEB-BASED MEDICAL SERVICES
}

\author{
Alexander Nikov ${ }^{1}$, Tramaine Gumaia ${ }^{2}$
}

\begin{abstract}
Emotional User Experience Design (eUXD) has become increasingly important for web-based services. The primary objective of this study is to enable users to use websites that are easy to understand and operate and pleasing to use. A checklist tool for an emotional user experience (eUX) assessment that supports web-based medical services is proposed. This tool measures user moods while using medical services' websites. The tool allocates emotive design-oriented problems and thus defines relevant website design recommendations. A case study was performed illustrating the proposed tool on a website offering medical services. Five variants of this website with different design element categories were created. These variants were assessed, analyzed, and proposed for further redesign in applying the tool. The results reveal the variant and website design elements that best influenced eUX. Significant improvement in eUX is expected with implementation of design recommendations of this study. The advantages of the tool are: (1) measuring emotional responses of medical website users, (2) allocating emotive problems, (3) defining of recommendations for eUX design of web-based medical services, and (4) creation of suitable practices for eUX design of web-based medical services.
\end{abstract}

UDC Classification: 617.7 DOI: http://dx.doi.org/10.12955/cbup.v4.862

Keywords: User experience, user emotions, assessment, tool, checklist, web-based medical services.

\section{Introduction}

Today, healthcare website users are more technologically savvy than users of 10 years ago, and tend to be more confident and curious about using the internet to learn about their health. Generally, usage of medical websites depends greatly on the user's interest in specific medical information. Different groups of users include: health professionals (doctors and medical staff), acute or chronic patients, relatives of patients, and persons who seeking education on health including medical services and insurance. Although the internet will never replace the care of a healthcare professional, users acknowledge that it is as a vital place to start learning about their medical treatment and provides an effective tool for learning more about health topics that may be of interest to them.

Users justifiably, before visiting a website, have a wide range of emotions and for the most part these might be negative ones. In their search, website users might seek a result that offers comfort or reassurance. Users would also need to trust the information presented on a healthcare website. In the field of medical website design, it is important to have an interface that is not only efficient and effective in terms of usability, but is an emotional-oriented interface (Parthasarathy \& Fang, 2013). An appropriate website design that caters for both functional and emotional elements will enhance the image of doctors and other health professionals on the internet, as well as the medical facility. The design would also affect whether a user decides to remain on the website and whether they will make an appointment using the website.

Kansei Engineering (KE), first known as emotional engineering, was developed by Professor Nagamagai of Hiroshima University (Chen, 2013). The focus of KE was to identify the Kansei value of products that trigger an emotional response (Lokman, Noor, \& Nagamachi, 2009). Website developers can apply this technology to website design. It is a theory based on the user's emotional needs and therefore provides a user-oriented design philosophy through considering a user's emotional sense of the web interface.

Healthcare websites designed around intuitive images can be very effective. A good image or video animation can create a positive emotional connection for patients visiting the site. Color is subtle, but highly effective in invoking emotions (Jiang, Feng, Liu, \& Liu, 2008). It is important to choose colors that express a positive emotion, for example, a healthcare website could choose from a palette of warm natural colors to suggest the warmth of the patient-physician relationship (Kim, Lee, \& Choi, 2003).

\footnotetext{
${ }^{1}$ Alexander Nikov, Faculty of Science and Technology, The University of the West Indies, St. Augustine, Trinidad and Tobago, alexander.nikov@sta.uwi.edu

${ }^{2}$ Tramaine Alaina Gumaia, Caribbean Industrial Research Insitute (CARIRI), Trinidad and Tobago, tgumaia@gmail.com
} 
This article presents a checklist tool for emotional user experience (eUX) assessment, to help evoke a positive emotional experience of web-based medical services. This tool assists in measuring emotional responses, allocating emotive problems, and defining recommendations for eUX design of web-based medical services. A case study implementing this tool is presented and relevant redesign guidelines generated.

\section{Checklist tool for eUX assessment}

A checklist tool for eUX assessment of web-based medical services is proposed. Five steps of performing eUX assessment are involved in the checklist tool (Figure 1). In step 1, website design elements and their categories (Figure 2) are determined. At step 2, Kansei word pairs for measuring emotional user experience are selected (Figure 3). At step 3, user testing tasks are defined. These are typical tasks a user would perform on website variants in a "real-life" scenario. At step 4, the emotional user experience with a website variant is assessed by Kansei word pairs on the scale of 1 to 5 (Figure 3). This scale is from 1 (effective) to 5 (poor). A partial least squares (PLS) regression analysis was used to analyze the data. It used controllable (independent) variables (design elements) to explain or predict user emotional response (dependent) variables (Kansei word pairs). The PLS regression analysis constructs predictive models in case of many highly collinear factors (Haenlein \& Kaplan, 2004). By standardized regression coefficients, the relative strengths of the coefficients were compared. Each Kansei word pair had means and a range, and where the mean value was larger than the range, the design element was considered to have effective emotional influence on the website design. At step 5, based on results of step 4, eUX problems were allocated and relevant website redesign guidelines and recommendations generated.

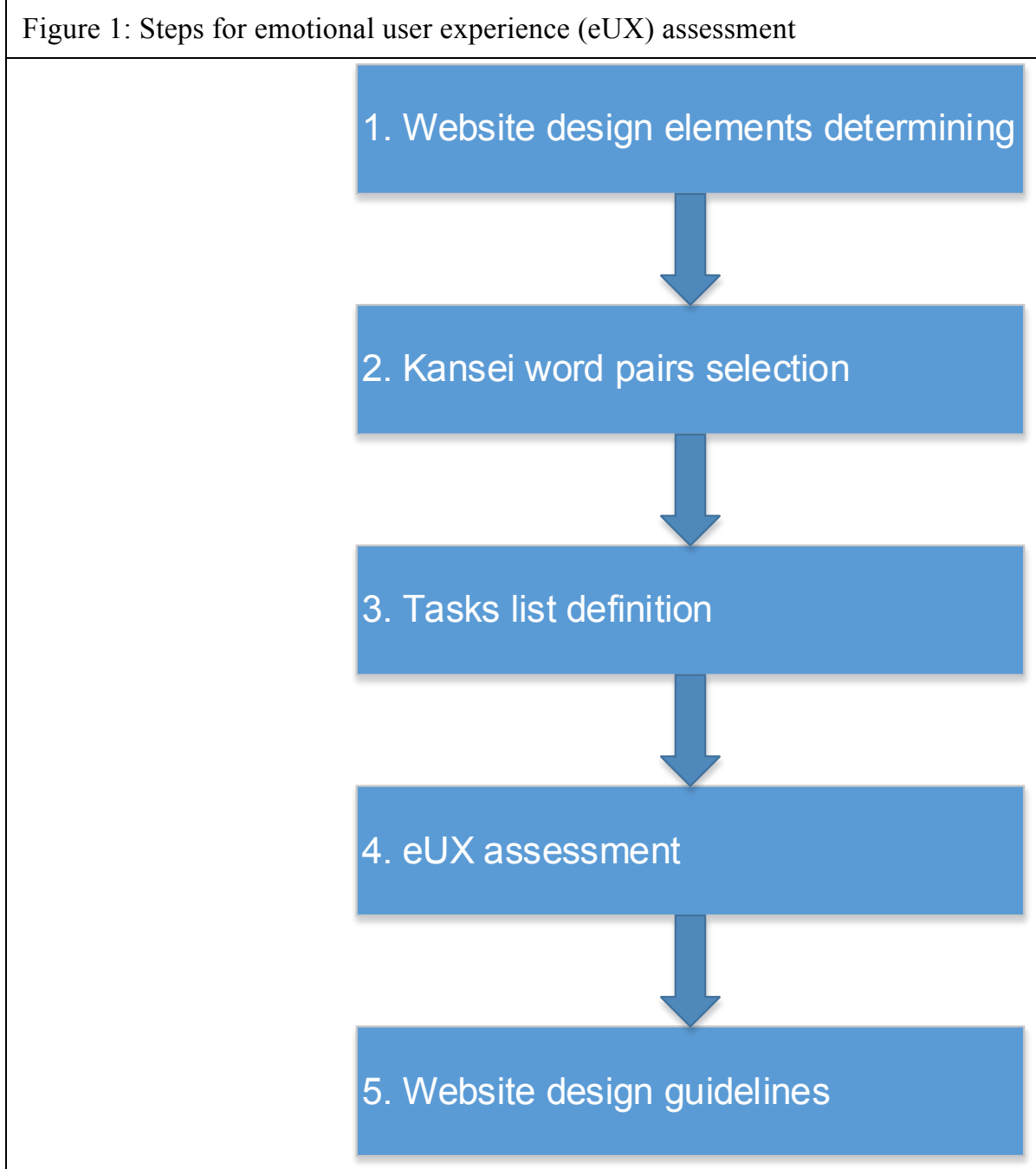

Source: Authors 
Figure 2: Fragment of website design elements and their categories

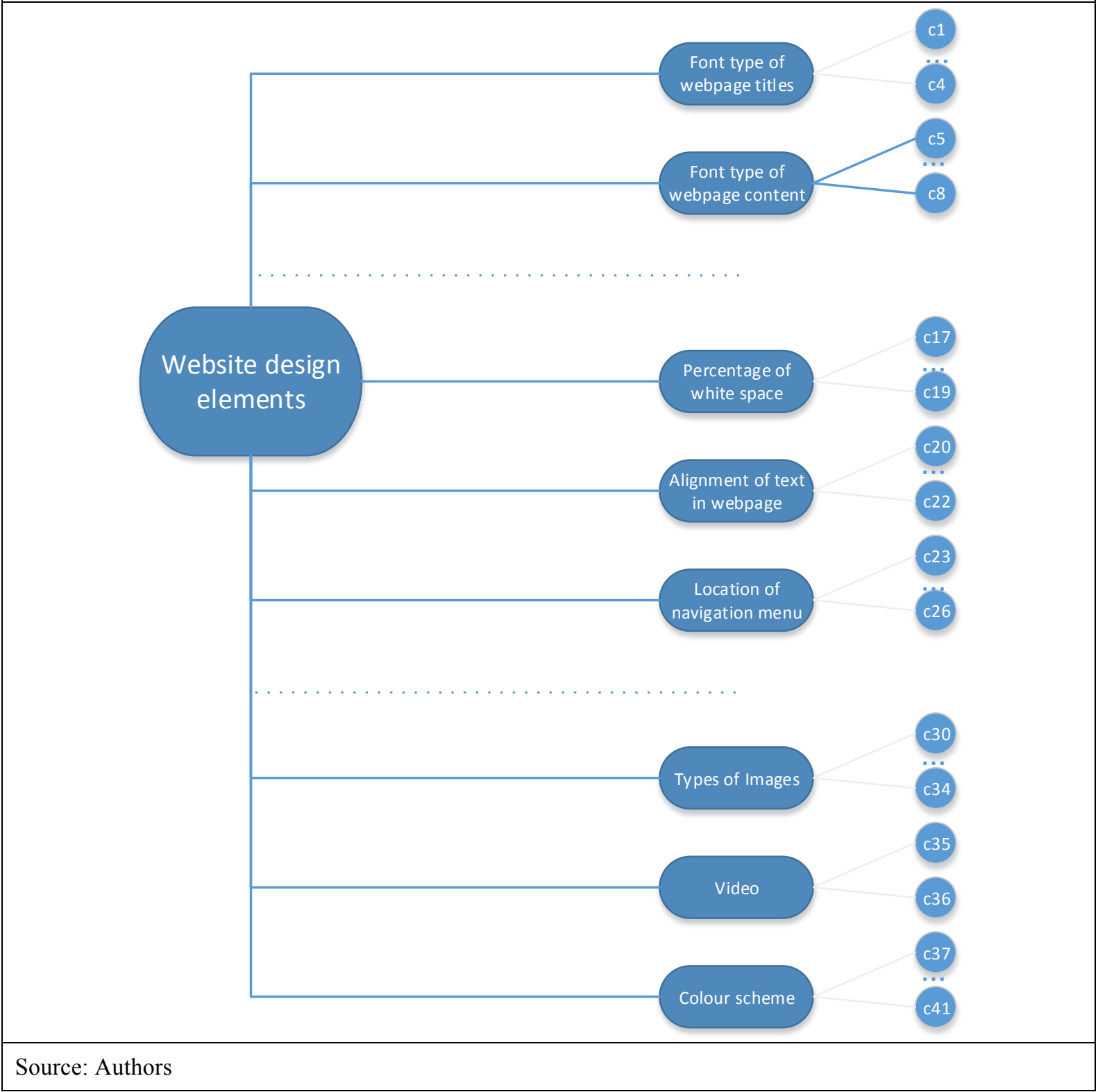

Figure 3: Example of Kansei word pairs and their measuring scale

\begin{tabular}{|l|l|l|l|l|}
\hline 1 & 2 & 3 & 4 & 5 \\
\hline
\end{tabular}

Good Design

Bad Design

\begin{tabular}{|l|l|l|l|l|}
\hline 1 & 2 & 3 & 4 & 5 \\
\hline
\end{tabular}

Like

Dislike

Source: Authors 


\section{Case study}

A case study using the checklist tool was performed. Patients and doctors (39 males and 33 females; total 72) ranging from ages 19 to 55 years, were surveyed to investigate eUX for five medical services website variants. The Variants 1 to 3 showed $100 \%$ task completion (Figure 4). Variant 5 had the lowest effectiveness, with $80 \%$ (Figure 4). The results show that Variant 3 had the best average for an eUX score (2), and therefore had the best user experience and was the most preferred (Figure 5).

Figure 4: Effectiveness of each website variant

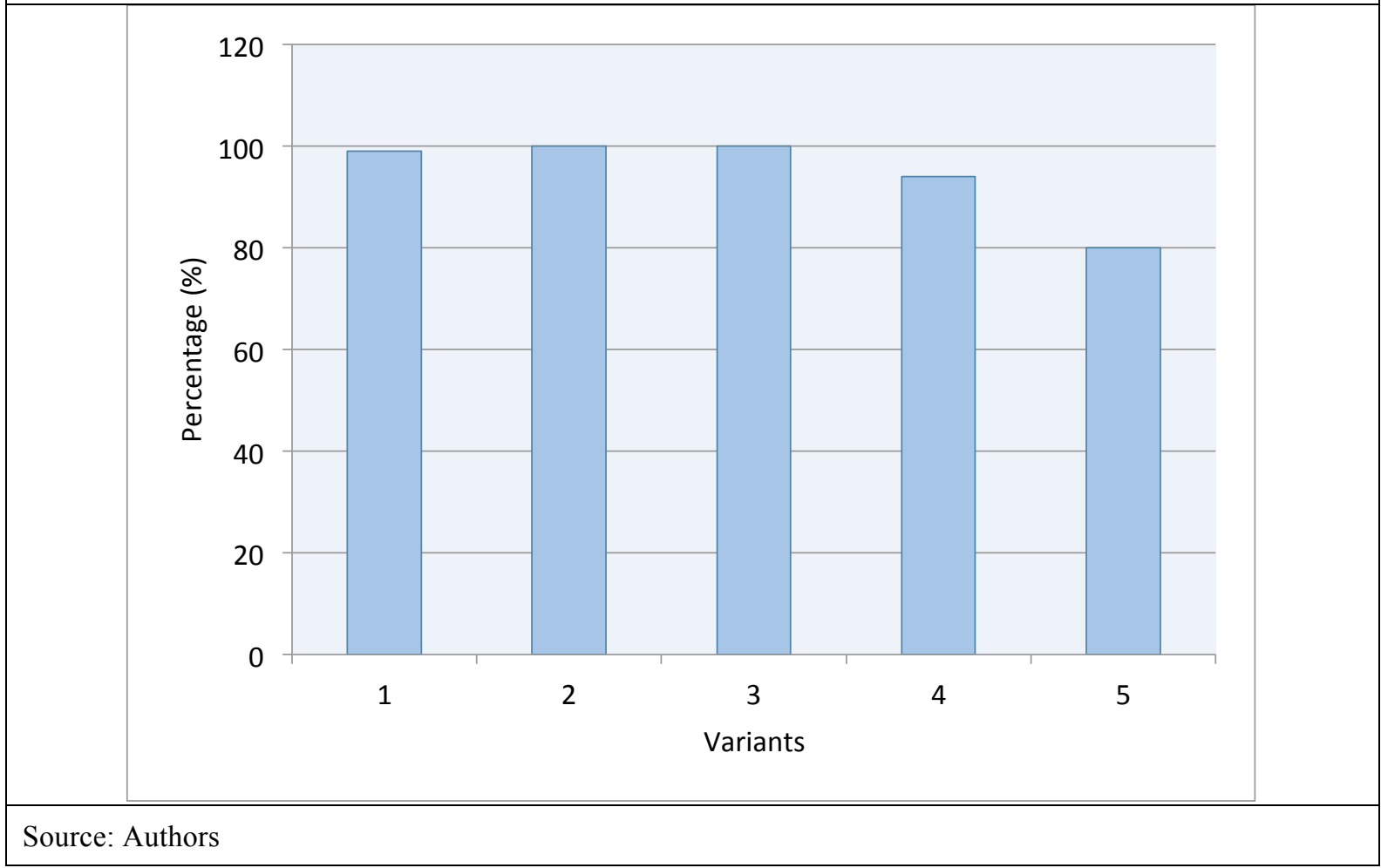

Figure 5: Average Kansei word pairs score for each website variant

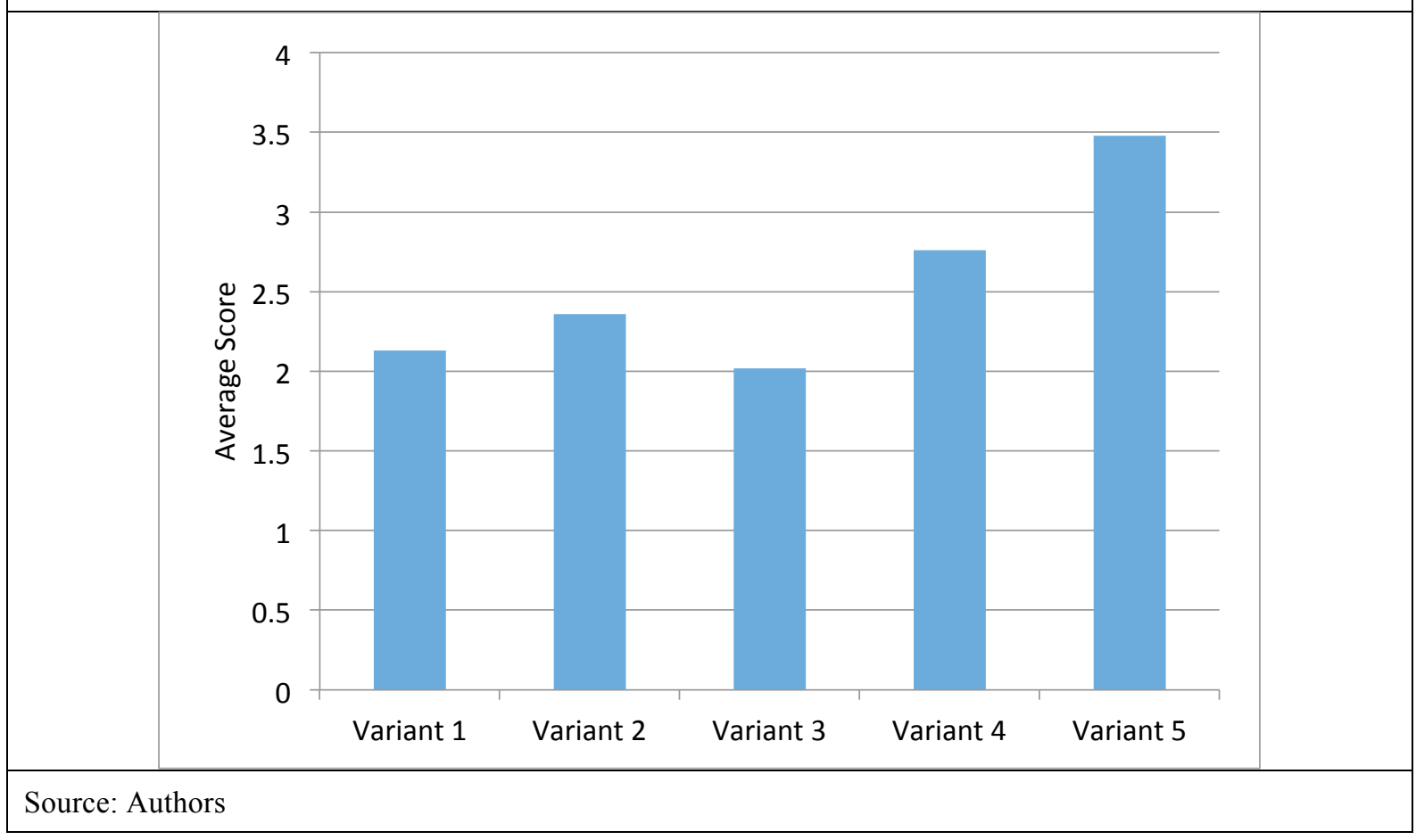


Table 1: Fragment of eUX assessment results and relevant redesign guidelines

\begin{tabular}{|l|r|c|c|}
\hline Design elements & Range (\%) & Effective design & Poor design \\
\hline Location of Logo & 20.8 & Left & Centre \\
\hline No of columns & 16.8 & Two & One \\
\hline Colour scheme & 15.7 & Blue & Purple \\
\hline Font Type of Webpage Titles & 11.0 & Sans-Serif (Bold) & Sans-Serif (Reg) \\
\hline Alignment of Text in Webpage & 8.9 & Left & Right \\
\hline Video & 8.3 & Yes & No \\
\hline Font Type of Webpage Content & 8.2 & Serif & Sans-Serif \\
\hline & 10.3 & & \\
\hline Average & & \\
\hline
\end{tabular}

In terms of medical services' websites that convey effective emotional user experiences, the results showed that the location of a logo, number of columns, color scheme, and font type of webpage titles, had the most influence, with higher than average score ranges recorded, i.e., above 10.3 (Table 1). On this basis, website designers might consider the following factors when choosing their web-based medical services design:

1. Location of logo; users are likely to prefer the conventional placement of the logo at the topleft.

2. Number of columns; for layout of website content, users preferred the dual column as an effective design.

3. Color scheme; Color plays a significant role and is the element first apparent to a user visiting a website. The results showed users preferred the color blue for a medical services website.

4. Font type of webpage titles; this design element plays a significant part in the emotion that designers aim to invoke in the user. For medical services websites, users preferred the technical "feel" of a sans-serif font for the title.

5. Alignment of text in webpage; this is another significant element to consider in designing the layout of a website. Users preferred left-justified text as it was regarded the most legible.

6. Video; recent studies have shown that videos are more effective than text. This was also the case in our study, as users preferred the presence of videos on medical services websites.

7. Font type of webpage content; this is another element that plays a significant part in the emotion that designers aim to convey. In the case of medical services websites, users preferred the warm and personal "feel" of a serif font for webpage content.

8. Font size of webpage content; users preferred the size of webpage content to be large.

9. Images; images are very perceptive design elements and provide users with a better understanding through an exchange of information. For medical services websites, the users preferred images of humans.

10. White space; a medium whitespace area was preferred by users.

11. Font size of webpage title; users preferred the size of webpage title to be large.

12. Location of menu; this is an important element with regards to navigation design. Many users liked the hidden menu design. However, for "easy navigation", the conventional location at the top-centre of the website was preferred.

\section{Conclusions}

A checklist tool for eUX assessment that supports positive eUX with web-based medical services was developed. This tool measures user emotions while they use medical services websites. By investigating the emotional states associated with the design elements, potential eUX problems were indicated and relevant website redesign recommendations were defined. These guidelines recommended that website design elements and their categories aid improved user experience for webbased medical services. A case study illustrating the proposed tool with a medical services website was performed. Five website variants with different design elements categories were studied. These were assessed using the checklist tool to analyze performances, and further redesign recommendations were proposed. The results revealed the category of different website design elements that best 
influenced eUX. Significant improvement of eUX is expected after implementation of this study's design recommendations.

The advantages of the tool are: (1) measuring emotional responses of medical services website users, (2) allocating emotive problems, (3) defining recommendations for eUX design of web-based medical services, and (4) creating good practices for eUX design of web-based medical services.

Further research oriented towards dynamic emotional user experience design of web-based medical services, using user brain wave recording and analysis, is recommended, as proposed previously (Ramirez \& Vamvakousis, 2012).

\section{References}

Chen, Y. (2013, June). Research on Optimized Design of Kansei Engineering-Based Web Interface. In Computational and Information Sciences (ICCIS), 2013 Fifth International Conference on IEEE (pp. 1709-1712).

Haenlein, M., \& Kaplan, A. M. (2004). A beginner's guide to partial least squares analysis. Understanding statistics, 3(4), 283-297.

Jiang, N., Feng, X., Liu, H., \& Liu, J. (2008). Emotional design of web page. In Computer-Aided Industrial Design and Conceptual Design, 2008. CAID/CD 2008. 9th International Conference on IEEE (pp. 91-95).

Kim, J., Lee, J., \& Choi, D. (2003). Designing emotionally evocative homepages: an empirical study of the quantitative relations between design factors and emotional dimensions. International Journal of Human-Computer Studies, 59(6), 899940.

Lokman, A. M., Noor, N. L. M., \& Nagamachi, M. (2009). ExpertKanseiWeb: A tool to design Kansei website. In Enterprise Information Systems, Berlin: Springer (pp. 894-905).

Parthasarathy, R., \& Fang, X. (2013). Introducing emotional interfaces to healthcare systems. In Human-Computer Interaction. Applications and Services, Berlin: Springer (pp. 150-162).

Ramirez, R., \& Vamvakousis, Z. (2012). Detecting Emotion from EEG Signals Using the Emotive Epoc Device, In F.M. Zanzotto et al. (Eds.) Bran Informatics, Lecture Notes on Computer Science, Berlin: Springer, Vol. 7670 (pp. 175-184). 